\title{
Evaluating Frequency, Diagnostic Quality, and Cost of Lyme Borreliosis Testing in Germany: A Retrospective Model Analysis
}

\author{
I. Müller, ${ }^{1}$ M. H. Freitag, ${ }^{2}$ G. Poggensee, ${ }^{3}$ E. Scharnetzky, ${ }^{4}$ E. Straube, ${ }^{1}$ \\ Ch. Schoerner, ${ }^{1}$ H. Hlobil, ${ }^{1}$ H.-J. Hagedorn, ${ }^{1}$ G. Stanek, ${ }^{1}$ A. Schubert-Unkmeir, ${ }^{1}$ \\ D. E. Norris, ${ }^{5}$ J. Gensichen, ${ }^{2}$ and K.-P. Hunfeld ${ }^{1}$ \\ ${ }^{1}$ Bacteriologic Infection Serology Study Group of Germany (BISSGG), Central Laboratory, Institute for Laboratory Medicine, \\ Northwest Medical Centre, Academic Teaching Hospital, Medical Faculty, Johann Wolfgang Goethe-University, Steinbacher Hohl 2-26, \\ 60488 Frankfurt am Main, Germany \\ ${ }^{2}$ Department of General Practice and Family Medicine, Jena University Hospital, Bachstraße 18, 07743 Jena, Germany \\ ${ }^{3}$ Surveillance Unit, Department of Infectious Disease Epidemiology, Robert Koch-Institute, DGZ-Ring 1, 13086 Berlin, Germany \\ ${ }^{4}$ Deutsche Angestellten-Krankenkasse (DAK), Nagelsweg 27-31, 20097 Hamburg, Germany \\ ${ }^{5}$ Department of Molecular Microbiology and Immunology, Johns Hopkins Bloomberg School of Public Health, Baltimore, \\ North Wolfe Street, MD 21205, USA
}

Correspondence should be addressed to K.-P. Hunfeld, k.hunfeld@em.uni-frankfurt.de

Received 8 July 2011; Accepted 8 September 2011

Academic Editor: Piotr Lewczuk

Copyright $\odot 2012$ I. Müller et al. This is an open access article distributed under the Creative Commons Attribution License, which permits unrestricted use, distribution, and reproduction in any medium, provided the original work is properly cited.

\begin{abstract}
Background. Data on the economic impact of Lyme borreliosis (LB) on European health care systems is scarce. This project focused on the epidemiology and costs for laboratory testing in LB patients in Germany. Materials and Methods. We performed a sentinel analysis of epidemiological and medicoeconomic data for 2007 and 2008. Data was provided by a German statutory health insurance (DAK) company covering approx. 6.04 million members. In addition, the quality of diagnostic testing for LB in Germany was studied. Results. In 2007 and 2008, the incident diagnosis LB was coded on average for 15,742 out of 6.04 million insured members (0.26\%). 20,986 EIAs and 12,558 immunoblots were ordered annually for these patients. For all insured members in the outpatient sector, a total of 174,820 EIAs and 52,280 immunoblots were reimbursed annually to health care providers (cost: $2,600,850 €$ ). For Germany, the overall expected cost is estimated at $51,215,105 €$. However, proficiency testing data questioned test quality and standardization of diagnostic assays used. Conclusion. Findings from this study suggest ongoing issues related to care for LB and may help to improve future LB disease management.
\end{abstract}

\section{Introduction}

Lyme borreliosis (LB) is a vector-borne disease that is transmitted by ixodid ticks and is caused by the spirochetes of the Borrelia (B.) burgdorferi sensu lato (s.l.) complex. The 5 genospecies that are currently considered to be human pathogens are B. burgdorferi sensu stricto (s.s.), B. afzelii, $B$. garinii, B. spielmanii, and the proposed but not yet validated novel species $B$. bavariensis [1]. Over the last 20 years, LB has been recognized as a major public health problem in the United States and in Europe [2,3]. Part of this status is related to variation in symptoms and the clinical picture of the disease [4]. In addition, further insecurity exists with the differential diagnostic considerations in LB patients, the natural trajectory of treated and untreated LB, and interpretation of diagnostic test results $[2,5,6]$. In Europe, the incidence of Lyme borreliosis is estimated to range from 0.6 to $155 / 100,000[4,7]$. In Germany, the estimation of 60,000-100,000 incident cases per year is based on an older seroprevalence study in a single region [8]. Mandatory reporting of new LB cases was established in 2002, but only in the six new federal states of the northeastern part of 
Germany. About 5,221 new cases of LB are reported for these states per year [9]. Little is known about the true incidence and distribution of LB in the other parts of Germany.

LB can manifest itself progressively as a multisystem disorder exhibiting a broad spectrum of clinical symptoms $[4,10]$. The disease is usually diagnosed clinically based on a characteristic clinical picture, a history of tick bite, and the diagnosis then can be supported further by serological testing. However, both false negative and false positive serologic test results do occur, and together with a lack of standardization of current diagnostic methods can clearly impede a clear and concise diagnosis [11]. Moreover, current law in most European countries does not require profound clinical evaluation of such commercially available diagnostic test kits for LB prior to market registration. Most significant, however, is the high seroprevalence of anti-B. burgdorferi antibodies that correlates with manifest disease in only a minority of patients. Therefore, serology should only be used to confirm but not to primarily establish the diagnosis of LB. In addition, the antibody titers on followups do not reflect the success of antibiotic treatment [12]. These factors can lead to misdiagnosis and mismanagement of LB and patients after tick bites and/or unspecific symptoms. As reported in the US, these events may lead to inappropriate care for patients including adverse effects and unnecessary financial cost $[2,5]$.

The situation in Germany is less obvious. Currently, little data is available on health care utilization in Germany for patients with confirmed or suspected LB such as performance of diagnostic and therapeutic measures including those for unspecific chronic conditions which are attributed to LB by patients or their physicians. The aim of the German investigation on Lyme borreliosis: evaluation of therapeutic and diagnostic cost (GILEAD) study is a step-wise analytic approach to estimate the amount of diagnostic testing, assay quality, and cost related to manifest and suspected LB in Germany. In this analysis, we explore the relative frequency of diagnostic testing, the number of incident and prevalent diagnoses, and the cost of laboratory diagnostics by analyzing German health insurance claims' data. In addition, we performed a meta-analysis of results obtained during the regular German LB serology proficiency testing program to learn more about the accuracy and reliability of currently used serological tests in Germany. This was done to evaluate the hypothesis that, although guidelines for the diagnostic management of LB with well-defined diagnostic algorithms for suspected LB cases have been established in Germany [13-15], relatively high volumes of diagnostic efforts (i.e., serologic testing) related to LB are being performed repeatedly without necessarily adding any benefit to the management of such patients.

\section{Materials and Methods}

2.1. Analysis of Health Insurance Datasets. The basic dataset consists of health insurance data from a German statutory health insurance company (Deutsche Angestellten-Krankenkasse, DAK) which covers approx. 6.04 million individuals all over Germany. The population insured by DAK consists of more women than men (ratio $1.8: 1$ ). In a first step, relevant international classification of diseases (ICD 10GM, 2004) diagnoses for Lyme borreliosis were defined as follows: ICD A69.2 for Lyme-specific erythema chronicum migrans, GO1* for LB-related meningitis, G63.0 for LBrelated polyneuropathy, and M01.2 for LB-related Arthritis. Claims' data of the years 2007 and 2008 were derived from the underlying datasets (patient data, ambulatory treatment data, and medication data). In accordance with legal data protection requirements, all personal data were exclusively handled by DAK. Analyses were based on subject-specific data which did not allow the identification of individual persons. Informed consent is not required for these analyses in Germany. The quality of the data was checked for completeness, correct usage of inclusion criteria, and plausibility prior to analysis according to existing standards [16, 17]. Individuals insured at least since January 1, 2006, or January 1, 2007, respectively, in whom at least one laboratory diagnostic procedure performed for LB in either year 2007 or 2008, were included in our analyses. The diagnostic procedures according to the general laboratory health insurance claim code ("Einheitlicher Bewertungsmassstab", EBM [18]) included laboratory claim numbers 32586 (B. burgdorferi antibody/enzyme-linked immune assay, ELISA), 32662 ( $B$. burgdorferi antibody/western blot), and/or 32743 (culture of B. burgdorferi). Individuals already having a coded diagnosis of Lyme borreliosis in 2006 were excluded from the analysis. We also extracted patient data (subject specifier, gender, year of birth, code for current residence, date of begin and end of insurance), ambulatory treatment data (subject specifier, quarter of the year, start and end of treatment period, diagnoses and diagnoses' assurance level, EBM codes, EBM date, area of specialty of physician), and medication data (subject specifier, date of prescription, agent, amount prescribed, cost).

\subsection{Collecting Data from the German Lyme Disease Proficiency} Testing Program. From March 2006 to November 2008, six LB serology proficiency testing surveys were conducted in Germany by the central reference laboratory for bacteriologic serodiagnostics of the Bacteriologic Infection Serology Study Group of Germany (BISSGG) now situated at the Institute of Laboratory Medicine, Northwest Medical Centre, Frankfurt/Main, in cooperation with the WHO Collaborating Centre for Quality Assurance and Standardization in Laboratory Medicine e.V. (INSTAND e.V.), Düsseldorf, and with the 9 reference laboratories of the BISSGG. The organisation and structure of the German proficiency testing program for bacteriologic infection serology is summarised elsewhere in more detail $[11,19,20]$.

2.3. Selection of Serum Samples. Twelve serum samples were obtained from voluntary donors according to previously published strict criteria and after obtaining written informed consent $[11,19]$. All subjects were clinically evaluated by experienced physicians. Six serum samples contained specific antibodies against $B$. burgdorferi as determined by various commercial test systems. All antibody-positive donors could recall a known history of a recent tick bite or active or past 
LB, which also had been documented in the medical records of these patients by their physicians. Six samples tested negative for specific antibodies against B. burgdorferi and were used as negative controls. Current or very recent LB was excluded in these donors by careful physical examination, evaluation of patients' medical histories, and review of the medical records provided by the referring physicians. Two of the six negative samples contained anti-T. pallidum antibodies and were obtained from a donor with a past syphilis infection and a donor eight weeks after appropriate therapy. Table 1 provides a detailed description of the clinical data available for all twelve samples.

\subsection{Study Conditions and Evaluation of Proficiency Testing} Results. Assessment of reference test results was performed according to the current guidelines of the German Medical Association and the standard operating procedures of INSTAND for proficiency testing in infection serology as recently described in more detail $[11,19]$. Qualitative and quantitative reference test results (Table 1) were determined for each pair of serum samples by 3 to 9 different specialised laboratories or university laboratories of the BISSGG with extensive expertise in the field of serodiagnostic testing for LB. Participation in the LB serology proficiency testing programs was not mandatory, but participating laboratories were required to register at INSTAND prior to their involvement. No preexisting criteria were established to exclude any laboratories from the survey. All participants were instructed to treat samples as routine samples and to perform their established serological test methods on the distributed samples blinded to additional clinical information to guarantee maximum objectivity. Qualitative and quantitative results had to be reported together with the methods used, the lot number and test manufacturer, and the laboratory machinery utilized [19]. Moreover, the laboratories reported interpretative statements as to whether the test constellation suggested a possible Borrelia infection and whether an early or late phase of infection was suspected. Reports were made in standardised form on defined evaluation sheets by use of a predefined code to permit statistical analysis after the surveys [19]. Only one test result per test method (indirect immune fluorescence assay: IFA, indirect hemagglutination assay: IHA, enzyme Immunoassay: EIA, chemoluminescence assay: CLIA, line blot, immunoblot, etc.) was reported to INSTAND by each participant. Participants were requested to return their reports to INSTAND for further computerassisted evaluation of results within 10 days after receipt of samples $[11,19]$. Qualitative results from participants were accepted as being accurate if their reported test results were congruent with the modal as determined by the reference laboratories (Table 1; Figure 1). Because the quantitative EIA results reported were so heterogeneous (Figure 2) owing to the different quantification methods of the test manufacturers, these results were not included in the evaluation listed below. Quantitative results of classical titre tests (IHA, IFA) were accepted as being accurate provided that results from participants were reported within a range of $\pm 2 \log _{2}$ unit dilutions around the median of the test results obtained by the reference laboratories. A qualifying certificate was forwarded to successfully participating laboratories for each parameter under the condition that their microbiological commentary, and qualitative and quantitative test results, for both samples using established assay systems met the abovelisted criteria $[11,19]$.

2.5. Statistical Analysis. All included claims' data were analyzed both within the entire group of individuals and within the group of patients with a coded incident diagnosis of A69.2 in the year 2007 or 2008 . To avoid misrepresentation, the population was analysed by age and gender and standardized with the general population of Germany (according to "Empfehlungen der Ersatzkassen und ihrer Verbände zur Umsetzung des §20 SGB V”). The numbers of all insured individuals were provided by gender, 5-year age groups, and 5-digit residence codes and used to compare to the group with a coded diagnosis of $\mathrm{LB}$ and/or a borreliosis test. Data analyses were descriptive and stratified by sex and standardized by age. Counts and incidence rates for LBrelated diagnostic testing were calculated. All data including proficiency testing results were reported as absolute numbers, means, modals, and percentages including standard deviations (SDs) as indicated and helpful (Figure 1). In addition, mean pass rates (Table 2; Figure 1) were calculated from the specific pass rates of the individual surveys performed biannually.

\section{Results}

3.1. Results from the German Proficiency Testing Program. From March 2006 to October 2008, between 360 and 392 microbiological laboratories (mean: $N=381, \mathrm{SD}=11$ ), including hospital laboratories, independent laboratories, physicians' office laboratories, and manufacturers of commercially available diagnostic LB assays took part in each of the six surveys that were held. Tests employed were those used routinely for the serodiagnosis of LB in the participating laboratories. Figure 1 gives an overview on the frequency of the various test systems used by the participants during the surveys. The laboratories most frequently performed a two-tier protocol, beginning with a sensitive EIA or CLIA screening (mean: $N=312(81.9 \%), \mathrm{SD}=6.9)$ followed by immunoblot or line blot confirmation of the results (mean: $N=282(74.0 \%), \mathrm{SD}=9)$, in compliance with the current recommendations of the CDC and most European scientific expert opinions $[13,21,22]$. On average, for direct immunoglobulin class-specific analysis of samples, IgGand/or IgM-EIA were used by $259(\mathrm{SD}=6)$ and 298 (SD = 9) of the participants, respectively, during the six surveys. An immunoblot confirmatory assay for IgG- and/or IgMantibodies was performed by $239(\mathrm{SD}=6)$ and $238(\mathrm{SD}: N=$ 7) of the laboratories, respectively. Some other traditional or more recently introduced serological test methods were employed much more rarely: IHA, mean: $N=15$ (3.9\%), IFA, mean: $N=23.5(6.2 \%)$, polyvalent EIA, mean: $N=33$ (8.7\%), CLIA, mean: $N=33(8.7 \%)$. Interestingly, there was a steady increase for new recombinant tests or hybrid assays (using native and/or recombinant protein preparations) and 


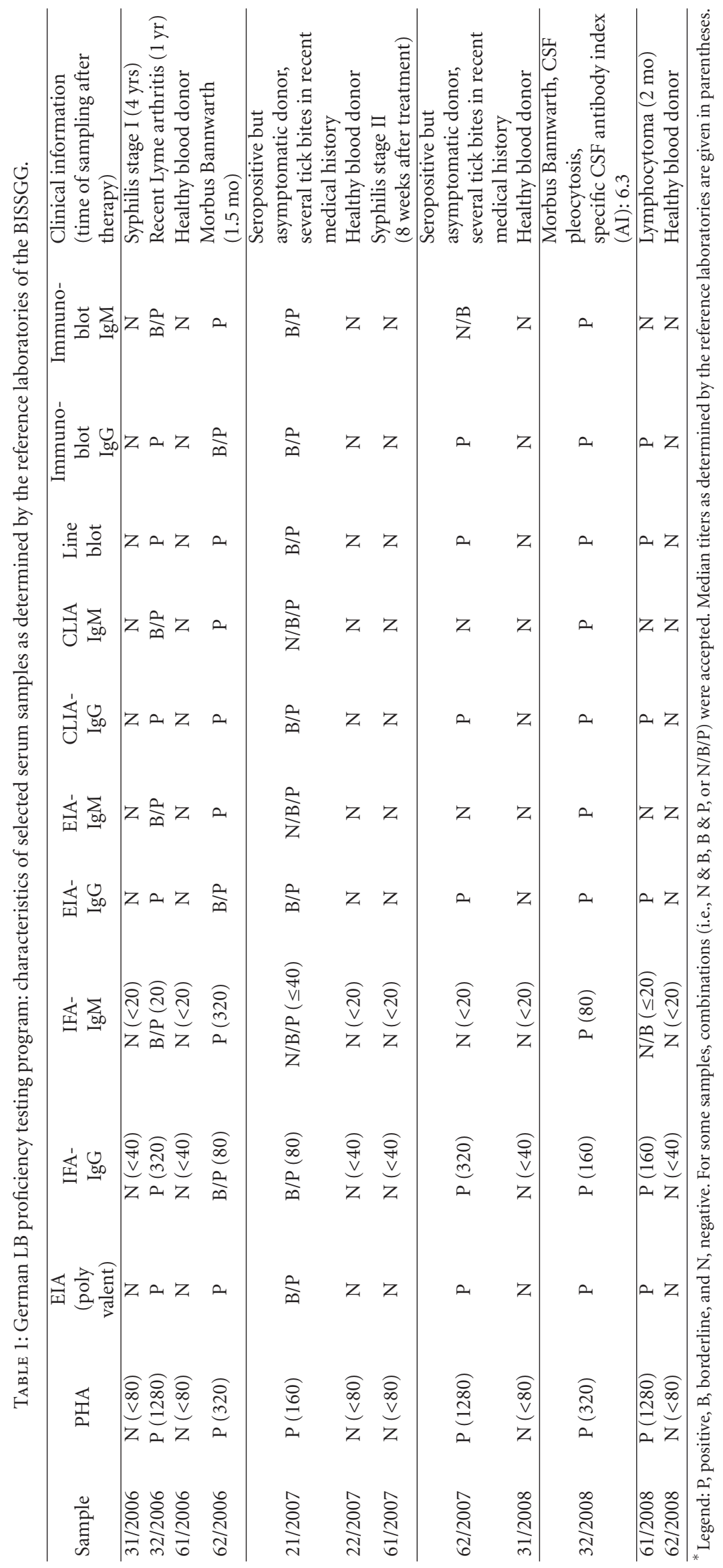




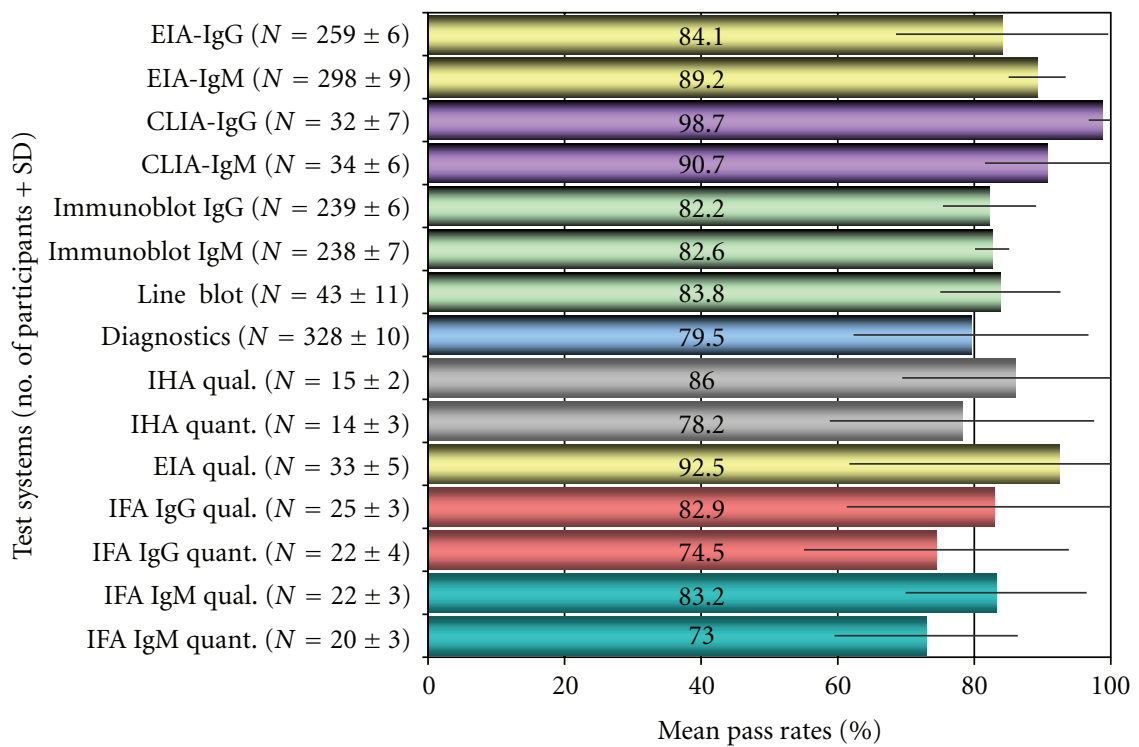

FIGURE 1: Average number of participants and mean pass rates (\%) with standard deviations (bars) for different assay systems as observed between 2006 and 2008 in the German LB proficiency testing program.

TABLE 2: Mean pass rates of proficiency testing participants in regard to samples tested and assay system used.

\begin{tabular}{|c|c|c|c|c|c|c|c|c|c|c|c|c|c|c|c|}
\hline Sample & & HA & $\begin{array}{c}\text { Polyvalent } \\
\text { EIA }\end{array}$ & $\begin{array}{l}\text { IgG- } \\
\text { EIA }\end{array}$ & $\begin{array}{l}\text { IgM- } \\
\text { EIA }\end{array}$ & $\operatorname{IgC}$ & -IFA & $\operatorname{IgN}$ & I-IFA & $\begin{array}{l}\text { IgG } \\
\text { Blot }\end{array}$ & $\begin{array}{l}\text { IgM } \\
\text { blot }\end{array}$ & $\begin{array}{l}\text { Line } \\
\text { blot }\end{array}$ & $\begin{array}{l}\text { IgG- } \\
\text { CLIA }\end{array}$ & $\begin{array}{l}\text { IgM- } \\
\text { CLIA }\end{array}$ & $\begin{array}{l}\text { Diagnostic } \\
\text { Comment }\end{array}$ \\
\hline $\begin{array}{l}\text { Positive } \\
\text { samples }\end{array}$ & Qual. & Quant. & Qual. & Qual. & Qual. & Qual. & Quant. & Qual. & Quant. & Qual. & Qual. & Qual. & Qual. & Qual. & Qual. \\
\hline $32 / 2006$ & 100 & 88.9 & 95.5 & 97.0 & 90.7 & 93.5 & 93.5 & 64.3 & 61.5 & 97.1 & 95.1 & 97 & 95.7 & 11.5 & 98 \\
\hline $62 / 2006$ & 66.7 & 76.9 & 82 & 83.3 & 92.0 & 88.0 & 85.7 & 90.9 & 78.9 & 89.2 & 89 & 83.3 & 100 & 71.4 & 88.7 \\
\hline $21 / 2007$ & 93.3 & 82.4 & 90.9 & 96.1 & 95.7 & 79.3 & 80.8 & 100 & 100 & 93.9 & 89.4 & 94.1 & 96.8 & 100 & 51.7 \\
\hline $62 / 2007$ & 85.7 & 66.7 & 91.4 & 99.2 & 93.7 & 95.8 & 81.8 & 71.4 & 75 & 100 & 84.9 & 96.9 & 100 & 91.2 & 97.7 \\
\hline $32 / 2008$ & 78.6 & 78.6 & 96.3 & 97 & 91.4 & 95.7 & 81 & 80 & 75 & 88 & 88.5 & 96.2 & 100 & 100 & 71.0 \\
\hline $61 / 2008$ & 100 & 100 & 96.2 & 99.6 & 81.4 & 90.9 & 77.3 & 89.5 & 78.9 & 100 & 87.7 & 96.7 & 100 & 91.8 & 96.5 \\
\hline Mean & 87.3 & 82.3 & 92.1 & 95.4 & 90.8 & 90.5 & 83.4 & 82.7 & 78.2 & 94.7 & 89.1 & 94.0 & 98.8 & 77.7 & 83.9 \\
\hline $\begin{array}{l}\text { Negative } \\
\text { samples }\end{array}$ & Qual. & Quant. & Qual. & Qual. & Qual. & Qual. & Quant. & Qual. & Quant. & Qual. & Qual. & Qual. & Qual. & Qual. & Qual. \\
\hline $31 / 2006$ & 94.1 & 88.9 & 95.9 & 93.9 & 95.5 & 93.5 & 93.5 & 85.7 & 84.6 & 97.9 & 94.6 & 93.9 & 100 & 100 & 98.3 \\
\hline $61 / 2006$ & 100 & 100 & 99.6 & 100 & 93.4 & 92.0 & 90.5 & 95.5 & 94.7 & 96.1 & 95.6 & 100 & 100 & 96.4 & 99.4 \\
\hline $22 / 2007$ & 100 & 100 & 87.9 & 88.8 & 93.7 & 62.1 & 53.8 & 92.6 & 92 & 84.6 & 90.2 & 75 & 100 & 100 & 92.4 \\
\hline $61 / 2007$ & 69.2 & 71.4 & 11.8 & 53.5 & 94.1 & 41.7 & 40.9 & 95.2 & 95 & 77.7 & 96.9 & 87.9 & 100 & 91.2 & 87.4 \\
\hline $31 / 2008$ & 100 & 100 & 96.2 & 98.9 & 96.4 & 91.3 & 90.5 & 100 & 95 & 94.4 & 98.3 & 100 & 100 & 97.4 & 99.4 \\
\hline $62 / 2008$ & 100 & 100 & 96 & 96.8 & 97.1 & 86.4 & 81 & 100 & 94.4 & 93.8 & 98.2 & 91.7 & 100 & 93.9 & 98.1 \\
\hline $\begin{array}{l}\text { Mean pass } \\
\text { rates }\end{array}$ & 93.8 & 93.3 & 81.2 & 88.6 & 95.0 & 77.8 & 75.0 & 94.8 & 92.6 & 90.7 & 95.6 & 91.4 & 100 & 96.5 & 95.8 \\
\hline
\end{tabular}

new analytical test formats such as CLIA and line blots, from $5.8 \%$ and $8.5 \%$ in 2006 to $10.5 \%$ and $16.4 \%$ in 2008 (data not shown).

3.2. Accuracy of Test Results. Characteristics of the selected serum samples applied in the German LD proficiency testing program as determined by the nine reference laboratories are depicted in Table 1. The percentages of laboratories that reported correct results with their routinely applied assay systems on the twelve serum samples sent out for testing in the six surveys of the German LD proficiency testing program from 2006-2008 are summarised in Table 2 and Figure 1, as individual pass rates per method and sample or as mean pass rates over time. IgG tests (mean pass rate: $92.1 \%$, range 41.7 to $100 \%$ ) were only slightly more accurate than those for IgM tests (mean pass rate: $90.3 \%$, range: 11.5 to $100 \%$ ). Mean pass rates for immunoblot testing (mean pass rates: $92.6 \%$, range: 75 to $100 \%$ ) were less accurate than those for 


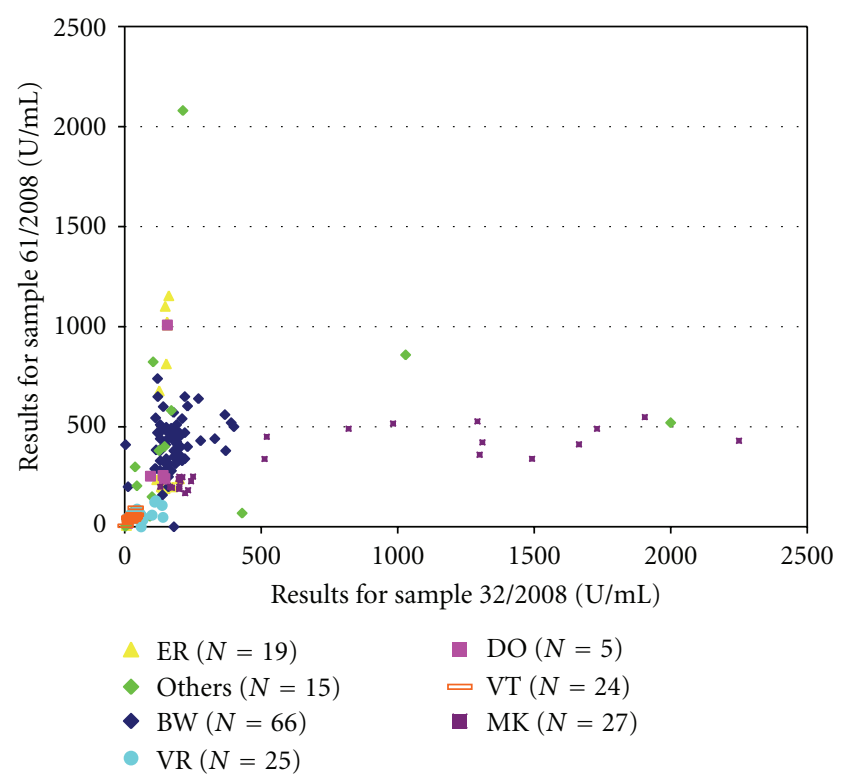

FIGURE 2: Youden's plot of different quantitative IgG-EIA results $(\mathrm{U} / \mathrm{mL})$ as obtained in two samples (no. 32 and 61 ) used for LB proficiency testing in 2008. For clinical information of samples, see Table 1. (ER, BW, DO, MK, VR, VT, others: anonymized abbreviations of different commercial EIA manufacturers).

EIA-testing (EIA mean pass rates: 92.5\%, range 53.5-100\%). Less frequently used tests such as polyvalent EIA, IFA, and IHA demonstrated mean pass rates for qualitative test results in the range of 11.8 to $99.6 \%$ (mean: $86.6 \%$ ), 41.7 to $100 \%$ (mean pass rates: $86.5 \%$ ), and 66.7 to $100 \%$ (mean pass rate: $90.6 \%$ ). Newly introduced test systems such as CLIA and line blot showed a variable performance with pass rates from 11.5 to $100 \%$ (mean pass rate: $89.0 \%$ ).

Serum samples from patients with long-lasting immune responses (Lyme arthritis, lymphocytoma, recent recurrent tick bites) showed that predominant IgG responses (32/2006, $62 / 2007,61 / 2008)$ were reproducibly detected and correctly interpreted by most of participants. In contrast to this finding, samples from patients with shorter disease duration or lower titers of specific IgM and IgG antibodies, such as sera from neuroborreliosis cases (62/2006 and 32/2008), past infections (serum scar: sample 21/2007), and cross-reacting samples such as sera from syphilis patients (61/2007), posed more significant diagnostic problems. Here, especially IHA and IFA tended to fail in identifying these samples correctly (Tables 1 and 2).

Our observations with classical titer tests revealed that although the calculated median IFA and IHA titers of the reference laboratories and those of the participants in the majority of cases generally varied only for one to two $\log _{2}$ unit dilutions around the median; the ranges of titers in the group of participants revealed an enormous variability of test results (data not shown). Similarly, the quantitative results in EIAs demonstrated a very low level of interassay standardisation in all trials, resulting in a rather high heterogeneity of reported quantitative results (Figure 2).
This finding is largely due to methodological differences of commercially manufactured assays and the variable methods of quantification (values of optical density (OD), indices, titers, $\mathrm{U} / \mathrm{mL}$ ) used. Similar to previous investigations [11], we decided not to include quantitative EIA results in the final evaluation of the proficiency testing surveys because of the obvious lack of assay standardisation.

Qualitative immunoblot test results were reported by the participants in all surveys that were performed (Tables 1 and 2). In addition, the laboratories reported the number and category of the specific IgG and IgM bands observed in their immunoblots for each of the serum samples (Figures 3(a) and 3(b)). The immunoblot results of the participating laboratories, however, showed that the individual results of the participants were not comparable in regard to the category and number of bands or the combination of bands (Figures 3(a) and 3(b)). Despite the high variability of serological test results, the microbiological interpretation of the different test constellations as reported by the laboratories was relatively homogeneous. Most participants (mean pass rate: $89.1 \%$, range: 51.7 to $99.4 \%$ ) correctly reported interpretative statements (Figure 1, Table 2) as to whether or not the assay results suggested a possible borrelial infection and whether an early or late phase of the specific antibody response was suspected.

3.3. False Positive and False Negative Test Results and Evaluation of Test Kit Quality. While using a variety of in-house tests and commercially manufactured LB test kits for the serodiagnosis of LB, participants reported a high number of false positive and false negative test results during the individual surveys. For IHA, false negative results were found in up to 33.3 of the reports and false positive results were reported in up to $30.8 \%$ of the participants. For the IgMIFA, the rate of false negative results reached up to $35.7 \%$ and the rate of false positive results varied from 0 to $14.3 \%$ during our surveys. For the IgG-IFA, both false negative and false positive results were reported in up to 20.7 respective $58.3 \%$ of the participants. For polyvalent ELISA, the rate of false negative and false positive results ranged from 3.7 to $18 \%$ and from 0.4 to $88.2 \%$. Class-specific ELISA testing also produced false positive results in up to $6.6 \%$ of the IgM- and in up to $46.5 \%$ of the IgG-ELISA reports. For immunoblot testing, false negative results were detected in $0-12 \%$ of the IgG- and in $4.9-15.1 \%$ of the IgM-assay reports. False positive blot results were reported in 2.1 to $22.3 \%$ of the IgG tests and in 1.7 to $9.8 \%$ of the IgM immunoblots. As depicted in Table 2 and Figures 1 and 4, the overall performance of assays was in part highly variable during the surveys and more or less depended on the assay type and manufacturer of the diagnostic test as shown for EIA in Figures 2, 4(a) and 4(b).

3.4. Outpatient Data Analysis of Patients with a Coded Diagnosis of LB. Throughout the years 2007 and 2008, an average of 6,042,531 individuals (male: female ratio $1: 1.6$, average age: 54.7 years, male subgroup: 53.2 years, female subgroup: 55.5 years) were insured by the German health insurance provider "Deutsche Angestellten-Krankenkasse" (DAK). In the years 2007 and 2008, a total of 22,282 and 


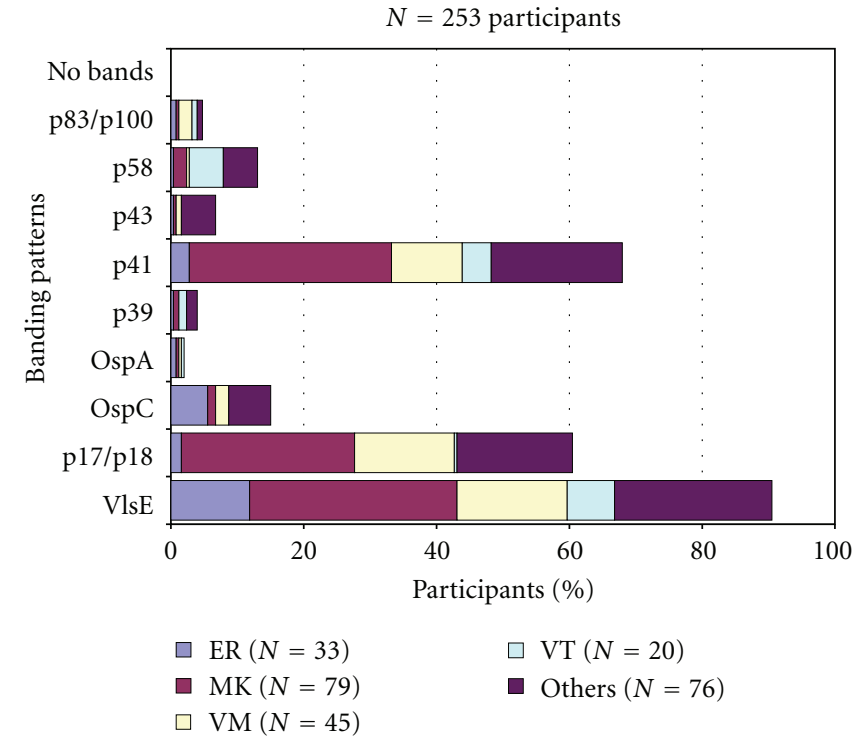

(a)

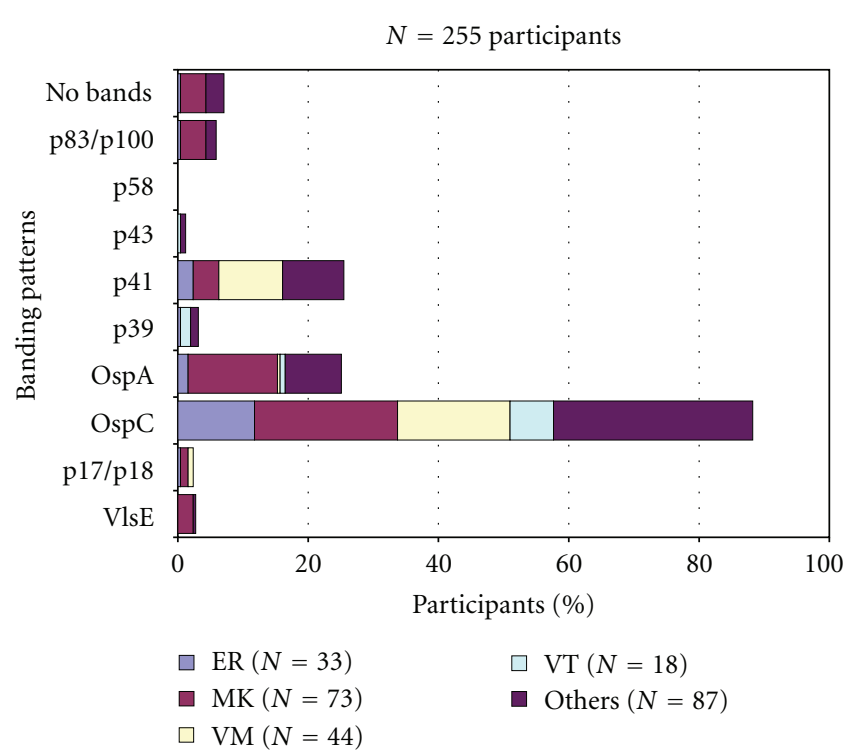

(b)

FIGURE 3: Recovery of LB-specific IgM- (a) and IgG- (b) immunoblot banding patterns (reported borrelial antigen bands: p83/100, p58, p43, p41, p39, OspA, OspC, p17/18, VlsE) as obtained from the participating laboratories for one sample (no. 32/2008) used for LB proficiency testing in 2008. Absolute frequency of participants reporting positive results for each antigen are depicted by bars. Relative frequency of positive reports for the different manufacturers are shown by colored boxes within the bars. (ER, MK, VM, VT, others: anonymized abbreviations of different commercial blot manufacturers). For clinical information of samples, see Table 1.

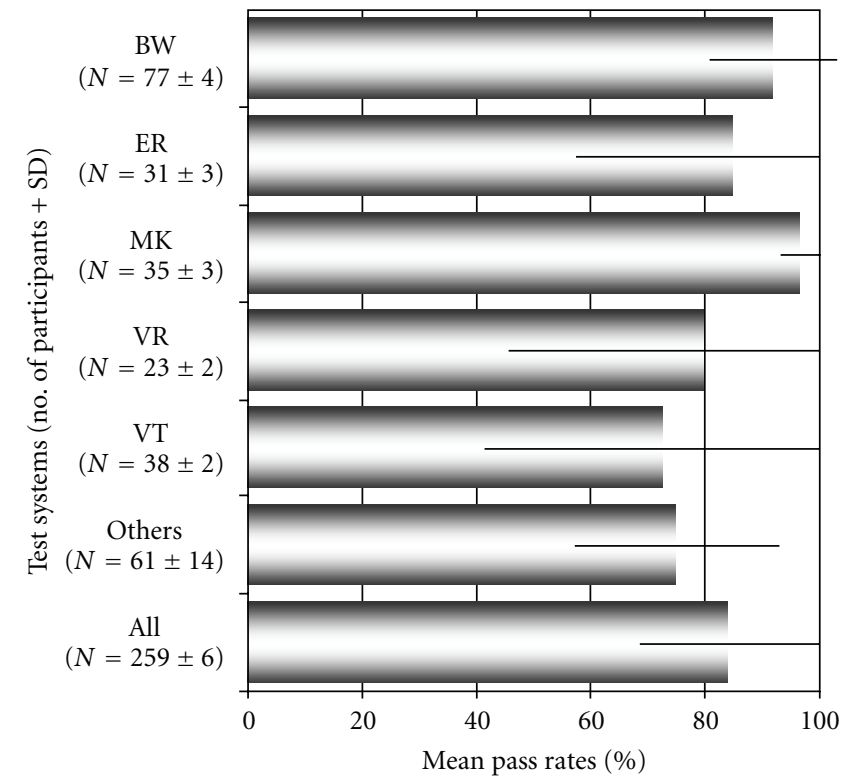

(a)

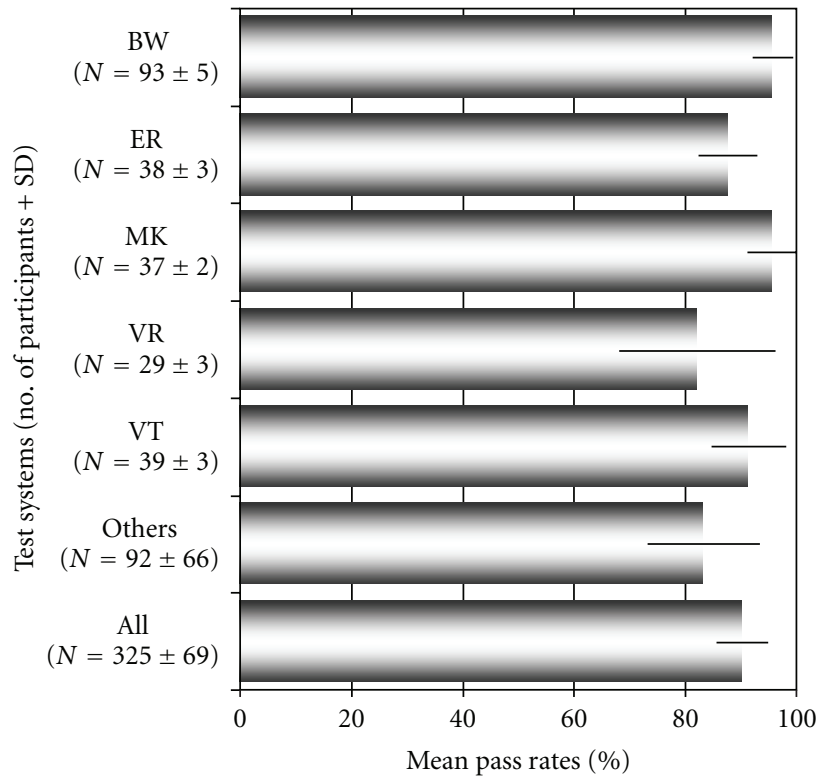

(b)

FIGURE 4: Average number of participants and average pass rates (\%) with standard deviations (bars) for IgG- (a) and IgM- (b) EIAs of different manufacturers as observed between 2006 and 2008 in the German LB proficiency testing program (ER, BW, DO, MK, VR, VT, others: anonymized abbreviations of different commercial EIA manufacturers).

25,184 DAK patients were diagnosed with $\mathrm{LB}$, respectively, which results in a prevalence of diagnoses of 365/100,000 $(0.37 \%)$ and $442 / 100,000(0.44 \%)$, respectively (average/ year: 404/100,000). Sixty-six percent of diagnosed patients were female, and $34 \%$ were male. The average age of the cohort was 52.4 years (range: 0-98 years). However, a new diagnosis of LB was coded only in a fraction of these patients. For our current analysis, an incident diagnosis of $\mathrm{LB}$ was accepted only for the investigated individuals if a past diagnosis of LB could be excluded for both 2006 
and in the preceding months of the analysed year 2007 and/or 2008. After exclusion of these (prevalent) diagnoses, 14,799 and 16,684 incident diagnoses remained for the years 2007 and 2008, respectively, leading to an average annual incidence for the diagnosis "LB" of 261/100,000 (diagnosis incidence of 242/100,000 and 279/100,000 individuals/year). Of these patients, 20,503 were female and 10,969 were male (m/f ratio: $1: 1.9)$. Average age was 50.2 years (range: 1-98 years) for males and 53.6 years (range: 0-96 years) for females. Figure 5 shows the distribution of incident and prevalent diagnoses throughout the two years analysed for this study, plotted against the data resulting from the mandatory reporting of incident LB cases for 2007 and 2008 for the eastern German states where mandatory reporting of LB is in place [9]. As depicted in Figure 5, the annual distribution of ambulatory LB diagnosis fits well into the epidemiological pattern known for incident cases from the data reported for the eastern German states [9]. Per annum, 9,303 of the newly coded patients were tested for LB serology leading to an average of 20,986 EIA tests and 12,558 immunoblots per year. Using diagnostic claims code data for these procedures, the testing resulted in a total amount of $462,980 €$ in diagnostic cost annually. Moreover, 19,683 antibiotic treatment courses were administered in these patients resulting in average costs of $563,508 €$ for antibiotic treatments per year.

3.5. Analysis of Sickness Fund Data on Laboratory Diagnostics in All Insured Individuals. In 2007, a total of 164,634 EIAs were ordered in 94,699 individuals. 27,362 individuals were tested further using 46,627 confirmatory immunoblots. Using diagnostic claims code data, the overall cost for serological LB diagnostics resulted in $1,267,681.8 €(7.7 € /$ EIA) for EIA and $1,119,048 €(24 € /$ test $)$ for confirmatory immunoblots. In 2008, a total of 185,007 EIAs and 57,934 immunoblots were performed in 112,150 and 35,002 individuals, respectively. Overall diagnostic cost in 2008 for serological diagnostics resulted in $1,424,554 €$ for EIAs and $1,390,416 €$ for western blotting. For the same time period, only 15 cultures of $B$. burgdorferi were claimed. In both years, the highest number of tests was performed in the 2nd and 3rd quarter of the year corresponding to the highest number of incident diagnoses (Figure 5). Assuming that our insurance sample $(7.4 \%$ of the German population) is representative for the whole German population of approximately 82 million [23], in the years 2007/2008 an average of 213,913 incident cases could have been expected, but 2,362,439 EIAs and 706,493 western blots would have been performed, leading to a cost of $35,146,617 €$ for diagnostic testing. Adjusting these cost for an additional add on of $6,106,627.94 €$ for the relatively higher reimbursement of laboratory cost (EIA: $23.46 €$, blot: $53.62 €$ ) for the known $10.5 \%$ of individuals with private health insurance the calculated average annual cost would be even higher $(41,253,240.24 €)$. Moreover, 283,912 treatments would have been administered resulting in $9,961,865 €$ for antibiotic therapies. When extrapolating the findings from our cohort to the German population as a whole, this rather conservative calculation would translate into testing of

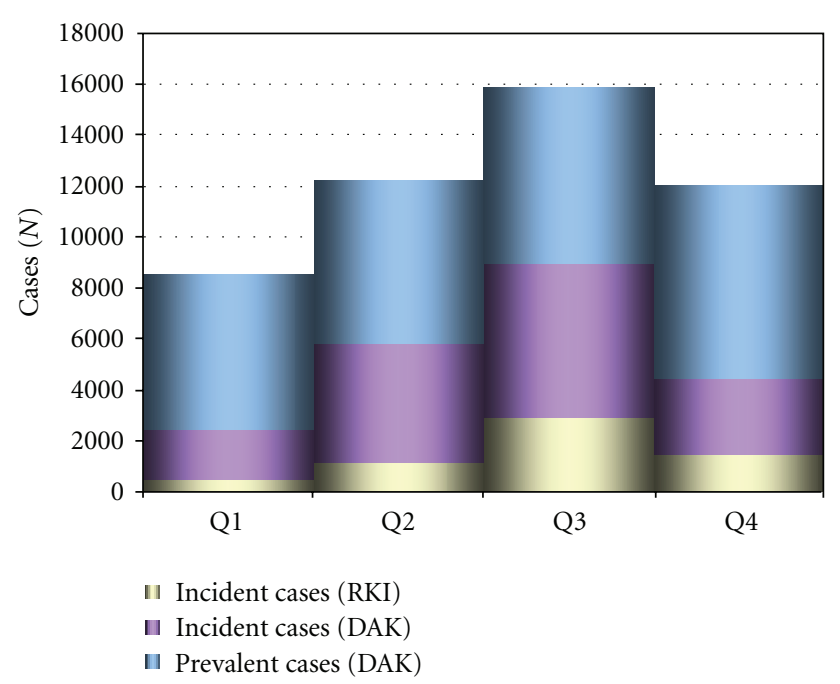

FIgURe 5: Average epidemiological annual distribution of coded incident and prevalent diagnoses of LB (incident DAK, prevalent DAK) as observed in the retrospective claims' data analysis depicted together with the average annual distribution of cases as reported by mandatory reporting (incident RKI) in the six new German states for 2007 to 2008. Q1-Q4: quarter of year.

$1,397,628$ individuals for suspected $\mathrm{LB}$ and total annual costs of $51,215,105.24 €$ for diagnostics and treatment. In contrast, focusing on incident LB cases, an average of 213,913 individuals would have been tested annually with 285,165 EIAs and 170,646 western blots leading to a cost of $6,291,290 €$ for diagnostic testing. Adjusting these cost for an additional add on of $1,002,617.17 €$ for the relatively higher reimbursement of laboratory cost (EIA: $23.46 €$, blot: $53.62 €$ ) for the known $10.5 \%$ of individuals with private health insurance would lead to average annual cost of $7,293,891.67 €$. Moreover, 237,000 treatments would have been administered resulting in another $7,614,973 €$ for antibiotic treatment. This calculation would translate to necessary annual costs of only $14,908,864.67 €$ for the diagnostics and treatment of incident LB patients in Germany, demonstrating a potentially significant gap between cost for indicated diagnostics and the high costs resulting from less selective healthcare services provided to the population as a whole.

3.6. Modelling the Influence of Diagnostic Test Quality and Cost on a Population-Based Scale. Applying available seroprevalence data from Germany [24-27] and our metaanalysed proficiency testing data to estimate the impact of the quality of diagnostic testing in Germany, we used mean pass rates for EIA and immunoblot as surrogate markers for average assay sensitivity and specificity. Given a projected number of 2,362,439 EIAs annually, we assumed these tests to be half IgG- and half IgM-specific tests. Assuming an average seroprevalence of $15 \%$ for Germany [24-27] and a mean IgG-EIA pass rate of $88.6 \%(\mathrm{SD}=16.1 \%)$ for negative and of $95.4 \%$ ( $\mathrm{SD}=5.5 \%$ ) for positive samples (see above), IgG screening in Germany would lead to approximately 114,460 false positive and 8,150 false negative test results. Using mean 
pass rates of $95.0 \%(\mathrm{SD}=4.4)$ for negative and $90.8 \%$ ( $\mathrm{SD}=$ 1.9) for positive samples for the performance of IgM-EIA, IgM screening would have resulted in 50,202 false positive and 16,301 false negative test results annually. Putting into use mean pass rates for IgG and IgM immunoblot of $90.7 \%$ $(\mathrm{SD}=7.2)(\mathrm{IgG}$ immunoblot $)$ and $95.6 \%(\mathrm{SD}=4.5)(\operatorname{IgM}$ immunoblot) for negative and of $94.7 \%(\mathrm{SD}=4.6)(\mathrm{IgG}$ immunoblot) and $89.1 \%(\mathrm{SD}=2.7)$ (IgM immunoblot) for positive samples, respectively, a given two-tier testing protocol would still result in 12,854 false positive and 26,495 false negative tests per annum on a population-based scale. In a different approach on a more test-specific basis, we also used our real world proficiency testing data (see above) to perform a fictive model calculation for a given two-tier testing protocol for specific IgG and IgM testing including computation of net sensitivities and net specificities. In the first model calculation, we used the mean proficiency testing pass rates for IgG- and IgM-ELISAs and immunoblots and obtained a net sensitivity of $90 \%$ for $\operatorname{IgG}$ and of $81 \%$ for $\operatorname{IgM}$ testing. The net specificity calculated for $98.9 \%$ for IgG and $99.8 \%$ for IgM.

In the second model calculation, we reduced the mean pass rates for IgG and IgM testing by the overall SDs as obtained throughout our surveys to adjust for slightly worse performing tests. This resulted in a loss of net sensitivity of $4.2 \%$ for IgM and of $9 \%$ for IgG and a loss of net specificity of $0.6 \%$ for IgM and of $3.4 \%$ for IgG. In this model, the reduction of net specificity and sensitivity led to an additional 192,716 immunoblot tests required and $4,625,183 €$ in additional cost. Moreover, the relatively small difference in net specificity added up to 6,191 additional false positive test results for IgM and 34,913 false positive test results for IgG. Finally, during our proficiency testing trials, we obtained a mean pass rate of $83.9 \%(\mathrm{SD}=17.2)$ in positive and $95.8 \%$ $(\mathrm{SD}=4.5)$ in negative samples for the correct diagnostic interpretation of laboratory test results. Given these pass rates, the serological testing of 1,397,628 individuals annually for $\mathrm{LB}$ as projected above would lead to a misinterpretation rate of up to $12 \%(N=167,715)$ even when it comes to the simple diagnostic question whether a positive or negative LB serology is present or whether an early or late phase of the antiborrelial immune response can be found.

\section{Discussion}

Similar to the situation in the United States with 20,000 30,000 LB cases reported annually [28], LB remains an important and very common indigenous infectious disease in Germany and Europe [8]. Although high morbidity can be expected from the disease due to the large number of cases and the potentially protracted course of the infection, little effort has been invested so far in investigations elucidating the epidemiological and financial impact of LB on the German health care system. This lack of health service data is striking when comparing LB with other common infectious diseases such as community-acquired pneumonia or nosocomial infections and is probably due to the readily available antimicrobial treatment options and the lack of mortality in LB. Likewise, such investigations are missing for most other parts of Europe making it difficult to assess the true dimension of the underlying medicoeconomical burden. Here, we used retrospective data analysis to examine (i) the epidemiology of LB, (ii) the quality of diagnostic testing, and (iii) the cost for diagnostic testing in Germany.

With official numbers absent for most parts of Germany, established mandatory reporting for 2007 and 2008 revealed about 5,624 annual incident cases (Figure 5) of LB (mainly erythema migrans) in the six new federal states (population: $16,507,263$ ) in the north-eastern part of Germany [9]. Extrapolating these findings to the German population of 82 million, this would translate into approximately 27,958 incident cases for the entire country annually. This strongly suggests significant underreporting even when compared to the commonly cited numbers of $60,000-100,000$ new LB cases per year as calculated from information available from previous seroprevalence investigations [8]. Thus, in a different approach, here, we used a retrospective outpatient data analysis performed between 2007 and 2008 on a cohort of 6,042,351 individuals insured by a German health care provider (DAK) to estimate the incidence and prevalence of LB in Germany by identifying the number of incident and prevalent diagnoses as a surrogate.

In the first step of our investigation, using these data, we were able to analyze a very large patient sample spread over the entire country and including all age groups. Importantly, possible bias due to recall, nonresponse, or the diagnostic process of attention is markedly reduced in such datasets compared to other study types [29]. Following strict definitions for the identification of "incident" and "prevalent" diagnoses, we identified 23,733 patients with a coded diagnosis of LB per year (prevalence: 404/100,000). An incident diagnosis of LB was coded in 14,799 and 16,684 individuals for the years 2007 and 2008, respectively, resulting in an incidence of 261/100,000 cases annually in the DAK cohort. Although the extrapolation of these numbers may lead to an overestimation due to clinical misdiagnosis and/or miscoding, our findings translate into 213,912 annual incident cases on a population-wide scale, which suggests more LB cases in Germany than projected previously in the available literature dealing with this topic.

Several recently performed interlaboratory studies have compared the diagnostic performance of serological tests for $\operatorname{LB}[5,11,30-35]$. Such investigations, however, can provide only limited information on the overall performance and relative accuracy of diagnostic testing in general on a nationwide scale. Therefore, in a second step our study was aimed at supplying additional data on the quality of LB diagnostics at the national level over a well-defined period of time. In addition, we tried to identify limitations and overuse of current diagnostic approaches to LB over a well-defined period of time paralleling our retrospective patient data analysis as outlined above. According to most guidelines, LB serology should only be performed to support clinical diagnosis, not as a primary basis for making diagnostic and/or treatment decisions [4]. As shown in this investigation and previous studies, serologic testing can be flawed by problems with both sensitivity and specificity [11]. Not unexpectedly, different methodological approaches 
in themselves can result, to some extent, in substantial differences with regard to test quality. Currently, a large variety of serological tests for the detection of LB are available in the European market, supplied by an increasing number of manufacturers. In the United States, a complex regulatory system for new in vitro diagnostics is in place which requires the manufacturer to compare its product substantially against an established device that has already been cleared by the FDA [36, 37]. In Europe, the institution of the new European IVD directive in 2000 did not legally insist in extensive, independent, and continuous clinical evaluation of commercially available serological test kits for LB before placing in vitro diagnostic tests on the market [38]. Instead, quality standards for the production quality and safety are enforced for in vitro diagnostic tests in their intended use $[38,39]$, and; consequently, test remakes are increasingly pushed onto the market. This trend is also supported by our observation showing a steady increase $(\sim 50 \%)$ of new test formats such as line blots and CLIA during the study period in the years 2006-2008. Currently, at least 55 different companies provide diagnostic tests for LB in Germany alone. Therefore, routine evaluations of microbiological laboratories by external quality control measures for LB serology appear to be attractive datasets to learn more about the relative frequency of certain test applications (i.e., EIA and immunoblot) in the diagnostic market, the amount of test standardisation, and the quality of performance in diagnostic infection serology [11, 19]. From 2006 to 2009, in Germany, between 360 and 392 microbiological laboratories took part in our proficiency testing surveys. Similar to findings of other investigations, most laboratories still relied on two-tier testing with EIA and immunoblot throughout the study period. Although qualitative testing by EIA and immunoblot showed mean pass rate ranges from 82.2 to $89.2 \%$ (Figure 1), quantitative EIA results and analysis of immunoblot banding patterns, however, demonstrated a very low degree of interassay standardisation (Figures 2 and 3). In addition, as already described in prior studies $[11,34,35]$, a high number of both false negative and false positive test results became obvious from our surveys and was in part correlated with the diagnostic method, the manufacturer (Table 2, Figures 1 and 4), and the amount of specific antibodies present in different sera (Table 1). Our findings confirm the assumption that, in the routine laboratory, the quantity of detected antibody measured in titers or quantitative EIA results and, similarly, the number and category of specific immunoblot bands can vary greatly for the same sample. In addition, changes in qualitative and quantitative serologic test results may be misleading and can emerge simply by using different assay systems in different laboratories. As a consequence of the findings in this investigation and other recent studies, a more general implementation of diagnostic criteria for the interpretation of immunoblot results as suggested by expert recommendations [13] seems increasingly difficult in light of the relatively high assay variability (Figures 2 and 3). Most importantly, correlating the activity of LB and the success of subsequent therapies with quantitative serological testing as well as with qualitative changes in the test results as attempted by some physicians appears clearly unreliable. The extreme variability of test results reported by the participants in our surveys is concordant with the few available international studies on this topic $[11,35,40-42]$. To improve the value of LB serology in the routine microbiological laboratory, promotion of better interassay standardisation of the commercially available test kits is necessary [13, 35, 42] by implementing standards and procedures as suggested earlier by the Centers for Disease Control and Prevention (CDC) and the Association of State and Territorial Public Health Laboratory Directors (ASTPHLD) conference on the serological diagnosis of LB [43]. Most importantly, more detailed and independent clinical evaluation of assays should be legally required before placing such products on the market [19].

So far, very few studies have examined the economic impact of quality and frequency of LB diagnostic testing on health care on the national level in the US and Europe. One study examined the medical and economical burden of LB in the United States. Using a decision analysis model and an estimated incidence of $4.7 \mathrm{LB}$ cases per 100,000 persons led to direct and indirect costs including diagnostic testing of about 2.5 billion US Dollars over 5 years for the US [44]. For the state of Maryland, Zhang et al. calculated direct medical costs including diagnostics of 2,970 US Dollars plus indirect costs of 5,202 US Dollars per LB patient [45]. This would add up to approx. 200 million US Dollars per year for the United States. Another study estimated the cost of LB for the Scottish health care system. Although this study was limited to laboratory testing only, the authors estimated a total of GBP 47,000615,000 for Scotland which seems to be a high financial impact for a country with a relatively low LB incidence [46]. All mentioned investigators used variable assumptions and economical models to assess the general cost of LB making it difficult to directly compare their findings to the results of our study. The results of these studies, therefore, cannot simply be transferred to the German health system due to differences in the epidemiology of the disease, of the methodological approaches, and in the health systems. Besides the cost for physician visits, consultation, and therapy, clearly, the cost for diagnostic testing, represents one of the major variables when calculating direct medical costs of LB on a population-wide scale. This is why, in the third step of our investigation, we tried to estimate the quantity and cost of diagnostic testing in Germany by modelling the combined information obtained from the DAK dataset and from the results of the regular German LB proficiency testing surveys run by INSTAND biannually to estimate the cost and medical quality of laboratory diagnostics on a nationwide level. By assessing the diagnostic frequency, quality, and cost of LB diagnostics in Germany, we estimated that 2,371,887 EIA tests and 709,331 western blots are performed annually. When modelling the influence of test quality for a given two-tier testing protocol including the calculation of net sensitivities and net specificities, it became obvious that using our real world proficiency testing data such tests would result in 12,854 false positive and 26,495 false negative test results annually on a population-wide scale. In this model, a small reduction in net specificity led 
to an additional 192,716 immunoblot tests required and an amount of $4,625,183 €$ in extra cost. Moreover, the small reduction in net specificity added up to 6,191 additional false positive test results for IgM and 34,913 false positive test results for IgG. Finally, given the average pass rates for the correct diagnostic interpretation of laboratory test results obtained during our proficiency testing trials, the serological testing of 1,397,628 individuals annually as projected above would lead to a misinterpretation rate of up to $12 \%$.

By further extrapolating the findings from our cohort of 6.04 million individuals insured by a statutory health insurance provider and adjusting our findings for $10,5 \%$ of privately insured patients with higher reimbursement, we project an overall expected cost of 51,215,105.24€ for LB serologic testing and treatment in Germany. These figures do not include patients' expenditure for nonrecommended tests (e.g., lymphocyte-transformation tests, LTT) which are not reimbursed but have to be paid out of the pocket. Similarly, in a study on LB management in primary care practices in Maryland USA, both diagnostic and therapeutic efforts were heavily overused [47]. In addition, Ramsey et al. showed that $80 \%$ of serology tests for Lyme borreliosis were regarded as inappropriate in a retrospective analysis in Wisconsin, USA [48]. Our findings on cost also come close to the study of Tugwell et al. who estimated 2.8 million tests in the US/year, leading to 100 million USD/year for serological testing [49]. The somewhat higher cost for testing in the US can be explained due to higher expenditure per test compared to the German situation. However, with our projected incidence of 214,000 cases per year, the overall frequency and cost of diagnostic testing and treatment clearly suggests a high amount of potentially inappropriate healthcare services in patients with a suspected or confirmed diagnosis of LB.

\section{Conclusion}

Our study is the first investigation of its kind in Germany and looks into the medical and economical burden of LB testing for the German healthcare system. Although suggesting a high amount of inappropriate diagnostic healthcare services, our analysis also shows limitations as it is focused on retrospective investigations of proficiency testing surveys and secondary claims data including external quality control datasets, coded diagnoses, and diagnostic and therapeutic services relevant for physician claims. Given our secondary data study design, other potential biases which cannot be accounted for including missing information on services provided outside the statutory health insurance and absence of information related to care provided which does not lead to (additional) claim codes (i.e., multiple visits within a quarter) $[29,50]$. However, the findings coming from the GILEAD project are a first approximation of health care services provided related to LB. They will help to assess and better tailor the quality standards for diagnostic tests and the economics of current and future disease management and prevention programs for LB. Given the ongoing problems in Germany with the clinical management of $\mathrm{LB}$, it seems important to closely monitor and evaluate health care utilization patterns including diagnostics and treatment for
LB patients to both facilitate a better understanding of existing care and design intervention approaches to improve the clinical management for such patients.

\section{Acknowledgments}

This study was funded by a grant provided by INSTAND e.V., Düsseldorf, Germany. Part of the study was supported by a research grant of the German Federal Ministry of Education and Research (BMBF 01GY1137). I. Müller and M. H. Freitag contributed equally as first authors to the study.

\section{References}

[1] G. Margos, S. A. Vollmer, M. Cornet et al., "A new Borrelia species defined by multilocus sequence analysis of housekeeping genes," Applied and Environmental Microbiology, vol. 75, no. 16, pp. 5410-5416, 2009.

[2] L. H. Sigal, "The Lyme disease controversy: social and financial costs of misdiagnosis and mismanagement," Archives of Internal Medicine, vol. 156, no. 14, pp. 1493-1500, 1996.

[3] H. M. Feder Jr, B. J. Johnson, S. O’Connell et al., "A critical appraisal of "chronic lyme disease"," New England Journal of Medicine, vol. 357, no. 14, pp. 1422-1430, 2007.

[4] G. Stanek, V. Fingerle, K. P. Hunfeld et al., "Lyme borreliosis: clinical case definitions for diagnosis and management in Europe," Clinical Microbiology and Infection, vol. 17, no. 1, pp. 69-79, 2011.

[5] A. C. Steere, E. Taylor, G. L. McHugh, and E. L. Logigian, "The overdiagnosis of Lyme disease," Journal of the American Medical Association, vol. 269, no. 14, pp. 1812-1816, 1993.

[6] L. H. Sigal, "Pitfalls in the diagnosis and management of Lyme disease," Arthritis and Rheumatism, vol. 41, no. 2, pp. 195-204, 1998.

[7] E. Lindgren and T. G. Jaenson, Lyme Borreliosis in Europe: Influences of Climate and Climate Change, Epidemiology, Ecology and Adaptation Measures, World Health Organization, Copenhagen, Denmark, 2006.

[8] G. Poggensee, V. Fingerle, K. -P. Hunfeld et al., "LymeBorreliose: Forschungsbedarf und Forschungsansatze. Ergebnisse eines interdisziplinaren Expertentreffens am Robert Koch-Institut. [Lyme borreliosis: research gaps and research approaches. Results from an interdisciplinary expert meeting at the Robert Koch institute]," Bundesgesundheitsblatt Gesundheitsforschung - Gesundheitsschutz, vol. 51, no. 11, pp. 1329-1339, 2008.

[9] Robert Koch-Institut, "Lyme-borreliose: Analyse der gemeldeten Erkrankungsfälle der Jahre 2007 bis 2009 aus den sechs östlichen Bundesländern," Epidemiol Bulletin, vol. 12, pp. 101110, 2010.

[10] A. C. Steere, "Lyme disease," New England Journal of Medicine, vol. 345, no. 2, pp. 115-125, 2001.

[11] K.-P. Hunfeld, G. Stanek, E. Straube et al., "Quality of Lyme disease serology: lessons from the German proficiency testing program 1999-2001. A preliminary report," Wiener Klinische Wochenschrift, vol. 114, no. 13-14, pp. 591-600, 2002.

[12] S. L. Brown, S. L. Hansen, and J. J. Langone, "Role of serology in the diagnosis of lyme disease," Journal of the American Medical Association, vol. 282, no. 1, pp. 62-66, 1999.

[13] B. Wilske and V. Fingerle, "Diagnostik der Lyme-Borreliose. Wie Sie den Verdacht auf Borreliose erhärten. [Diagnosis of Lyme borreliosis. How to corroborate suspected borreliosis]," 
MMW-Fortschritte der Medizin, vol. 142, no. 15, pp. 28-31, 2000.

[14] Deutsche Dermatologische Gesellschaft, kutane Manifestationen der Lyme Borreliose, [cited June 13, 2011]. http://www .awmf.org/uploads/tx_szleitlinien/013-044.pdf.

[15] Deutsche Gesellschaft für Neurologie, "Neuroborreliose," in Leitlinien für Diagnostik und Therapie in der Neurologie; 4 überarbeitete Auflage, H.-C. Diener and N. Putzki, Eds., p. 654, Georg Thieme Verlag, Stuttgart, Germany, 2008.

[16] H. T. Sørensen, S. Sabroe, and J. Olsen, "A framework for evaluation of secondary data sources for epidemiological research," International Journal of Epidemiology, vol. 25, no. 2, pp. 435-442, 1996.

[17] A. G. Erhebung and N. von Sekundärdaten, "AG epidemiologische Methoden. Gute Praxis Sekundärdatenanalyse (GPS). Version 2," 2008, [cited June 13, 2011]. http://dgepi.de/pdf/ infoboard/stellungnahme/gps-version2-final.pdf.

[18] Kassenärztliche Bundesvereinigung, Einheitlicher Bewertungsmaßstab Version 2/2011, KBV Kassenärztliche Bundesvereinigung, Berlin, Germany, 2011.

[19] I. Müller, V. Brade, H. J. Hagedorn et al., "Is serological testing a reliable tool in laboratory diagnosis of syphilis? Metaanalysis of eight external quality control surveys performed by the German infection serology proficiency testing program," Journal of Clinical Microbiology, vol. 44, no. 4, pp. 1335-1341, 2006.

[20] I. Müller, S. Besier, G. Hintereder, V. Brade, and K.P. Hunfeld, "Zur Qualität der bakteriologischen Infektionsserologie in Deutschland: eine Metaanalyse der infektionsserologischen Ringversuche des Jahres 2006-Beitrag der Qualitätssicherungskommission der DGHM," GMS Z Forder Qualitatssich Med Lab, vol. 1, pp. 1-21, 2009.

[21] Centers for Disease Control and Prevention, "Recommendations for test performance and interpretation from the second national conference on serologic diagnosis of Lyme disease," Morbidity and Mortality Weekly Report (MMWR), vol. 44, no. 31, pp. 590-591, 1995.

[22] K.-P. Hunfeld and P. Kraiczy, "When is the best time to order a western blot and how should it be interpreted?" Current Problems in Dermatology, vol. 37, pp. 167-177, 2009.

[23] Statistisches Bundesamt, "Bevölkerungsstand," 2011, [cited June 13, 2011]. http://www.destatis.de/jetspeed/portal/cms/ Sites/destatis/Internet/DE/Navigation/Statistiken/Bevoelkerung/Bevoelkerungsstand/Bevoelkerungsstand.psml.

[24] K.-P. Hunfeld, M. Ernst, P. Zachary, B. Jaulhac, H. H. Sonneborn, and V. Brade, "Development and laboratory evaluation of a new recombinant ELISA for the serodiagnosis of Lyme disease," Wiener Klinische Wochenschrift, vol. 114, no. 13-14, pp. 580-585, 2002.

[25] K.-P. Hunfeld, A. Lambert, H. Kampen et al., "Seroprevalence of Babesia infections in humans exposed to ticks in midwestern Germany," Journal of Clinical Microbiology, vol. 40, no. 7, pp. 2431-2436, 2002.

[26] R. Oehme, K. Hartelt, H. Backe, S. Brockmann, and P. Kimmig, "Foci of tick-borne diseases in Southwest Germany," International Journal of Medical Microbiology, vol. 291, supplement 33, pp. 22-29, 2002.

[27] R. Woessner, B. C. Gaertner, M. T. Grauer et al., "Incidence and prevalence of infection with human granulocytic ehrlichiosis agent in Germany. A prospective study in young healthy subjects," Infection, vol. 29, no. 5, pp. 271-273, 2001.

[28] Centers for Disease Control and Prevention, "Reported cases of Lyme disease by Year, United States, 1995-2009,” 2011, [cited June 13, 2011]. http://www.cdc.gov/lyme/stats/chartstables/casesbyyear.html.

[29] I. Schubert, I. Koster, J. Kupper-Nybelen, and P. Ihle, "Versorgungsforschung mit GKV-Routinedaten. Nutzungsmöglichkeite versichertenbezogener Krankenkassendaten für Fragestellungen der Versorgungsforschung. [Health services research based on routine data generated by the SHI. Potential uses of health insurance fund data in health services research]," Bundesgesundheitsblatt Gesundheitsforschung-Gesundheitsschutz, vol. 51, no. 10, pp. 1095-1105, 2008.

[30] C. W. Hedberg, M. T. Osterholm, K. L. MacDonald, and K. E. White, "An interlaboratory study of antibody to Borrelia burgdorferi," Journal of Infectious Diseases, vol. 155, no. 6, pp. 1325-1327, 1987.

[31] B. S. Schwartz, M. D. Goldstein, J. M. Ribeiro, T. L. Schulze, and S. I. Shahied, "Antibody testing in Lyme disease. A comparison of results in four laboratories," Journal of the American Medical Association, vol. 262, no. 24, pp. 3431-3434, 1989.

[32] R. S. Lane, E. T. Lennette, and J. E. Madigan, "Interlaboratory and intralaboratory comparisons of indirect immunofluorescence assays for serodiagnosis of Lyme disease," Journal of Clinical Microbiology, vol. 28, no. 8, pp. 1774-1779, 1990.

[33] S. W. Luger and E. Krauss, "Serologic tests for Lyme disease. Interlaboratory variability," Archives of Internal Medicine, vol. 150, no. 4, pp. 761-763, 1990.

[34] L. L. Bakken, K. L. Case, S. M. Callister, N. J. Bourdeau, and R. F. Schell, "Performance of 45 laboratories participating in a proficiency testing program for Lyme disease serology," Journal of the American Medical Association, vol. 268, no. 7, pp. 891-895, 1992.

[35] L. L. Bakken, S. M. Callister, P. J. Wand, and R. F. Schell, "Interlaboratory comparison of test results for detection of Lyme disease by 516 participants in the Wisconsin State laboratory of hygiene/college of American pathologists proficiency testing program," Journal of Clinical Microbiology, vol. 35, no. 3, pp. 537-543, 1997.

[36] J. N. Gibbs, "Regulations and standards. ASRs: FDA issues final rule. IVD Technology," 1998, [cited June 13, 2011]. http:// www.ivdtechnology.com/article/asrs-fda-issues-final-rule.

[37] S. A. Larsen, B. M. Steiner, and A. H. Rudolph, "Laboratory diagnosis and interpretation of tests for syphilis," Clinical Microbiology Reviews, vol. 8, no. 1, pp. 1-21, 1995.

[38] Bundesministerium fuer Gesundheit, "Bekanntmachung (AKZ 117-456000-02/2) zur EG-Richtlinie über in vitro Diagnostika (98/79/EG)," Bundesgesetzbl, vol. 118, 12077 pages, 2000.

[39] J. F. Place, "The coming age of in vitro testing. IVD Technology," 2000, [cited June 13, 2011]. http://www.ivdtechnology.com/article/coming-age-vitro-testing.

[40] C. W. Hedberg and M. T. Osterholm, "Serologic tests for antibody to Borrelia burgdorferi. Another pandora's box for medicine?" Archives of Internal Medicine, vol. 150, no. 4, pp. 732-733, 1990.

[41] M. C. Tammemagi, J. W. Frank, M. Leblanc, H. Artsob, and D. L. Streiner, "Methodological issues in assessing reproducibility: a comparative study of various indices of reproducibility applied to repeat elisa serologic tests for Lyme disease," Journal of Clinical Epidemiology, vol. 48, no. 9, pp. 1123-1132, 1995.

[42] E. C. Guy, J. N. Robertson, M. Cimmino et al., "European interlaboratory comparison of Lyme borreliosis serology," Zentralblatt fur Bakteriologie, vol. 287, no. 3, pp. 241-247, 1998. 
[43] "Recommendations," in Proceedings of the 2nd National Conference on Serologic Diagnosis of Lyme Disease, Association of State and Territorial Public Health Laboratory Directors and Centers for Disease Control and Prevention, Eds., Association of State and Territorial Public Health Laboratory Directors, Dearborn, Mich, USA, 1995.

[44] E. Maes, P. Lecomte, and N. Ray, "A cost-of-illness study of lyme disease in the United States," Clinical Therapeutics, vol. 20, no. 5, pp. 993-1008, 1998.

[45] X. Zhang, M. I. Meltzer, C. A. Peña, A. B. Hopkins, L. Wroth, and A. D. Fix, "Economic impact of Lyme disease," Emerging Infectious Diseases, vol. 12, no. 4, pp. 653-660, 2006.

[46] A. W. Joss, M. M. Davidson, D. O. Ho-Yen, and A. Ludbrook, "Lyme disease-what is the cost for Scotland?" Public Health, vol. 117, no. 4, pp. 264-273, 2003.

[47] A. D. Fix, G. T. Strickland, and J. Grant, "Tick bites and lyme disease in an endemic: setting problematic use of serologic testing and prophylactic antibiotic therapy," Journal of the American Medical Association, vol. 279, no. 3, pp. 206-210, 1998.

[48] A. H. Ramsey, E. A. Belongia, P. H. Chyou, and J. P. Davis, "Appropriateness of lyme disease serologic testing," Annals of Family Medicine, vol. 2, no. 4, pp. 341-344, 2004.

[49] P. Tugwell, D. T. Dennis, A. Weinstein et al., "Laboratory evaluation in the diagnosis of Lyme disease," Annals of Internal Medicine, vol. 127, no. 12, pp. 1109-1123, 1997.

[50] W. Hoffmann, C. Bobrowski, and K. Fendrich, "Sekundardatenanalyse in der Versorgungsepidemiologie: Potenzial und Limitationen. [Secondary data analysis in the field of epidemiology of health care. Potential and limitations]," Bundesgesundheitsblatt Gesundheitsforschung-Gesundheitsschutz, vol. 51, no. 10, pp. 1193-1201, 2008. 


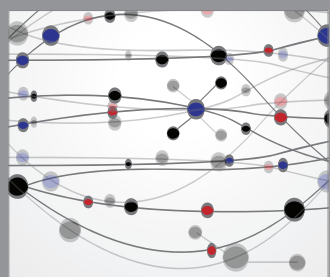

The Scientific World Journal
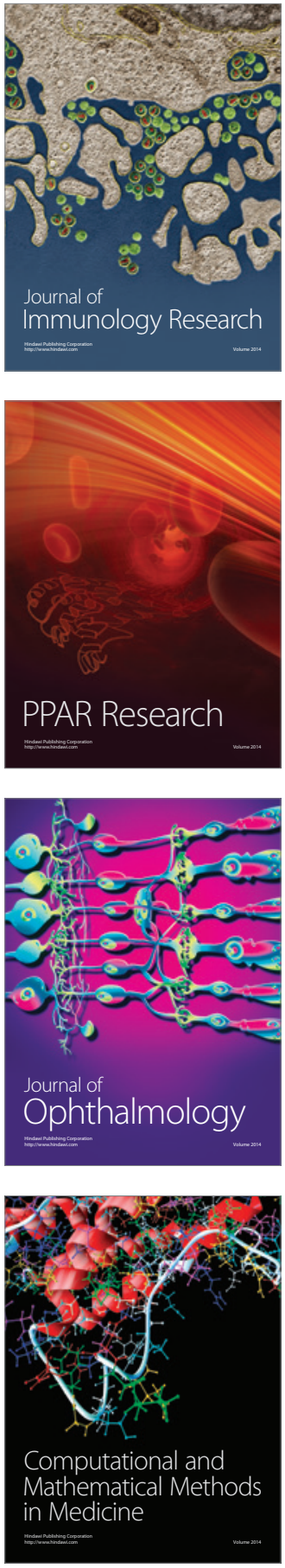

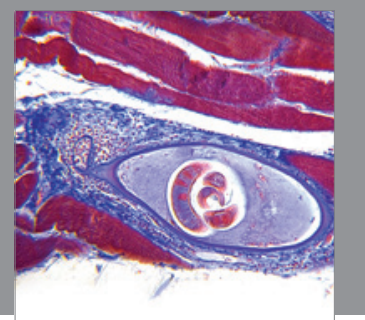

Gastroenterology

Research and Practice
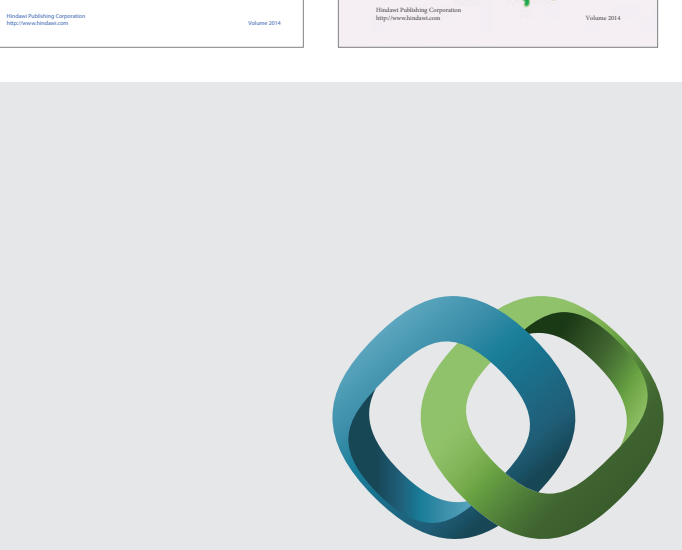

\section{Hindawi}

Submit your manuscripts at

http://www.hindawi.com
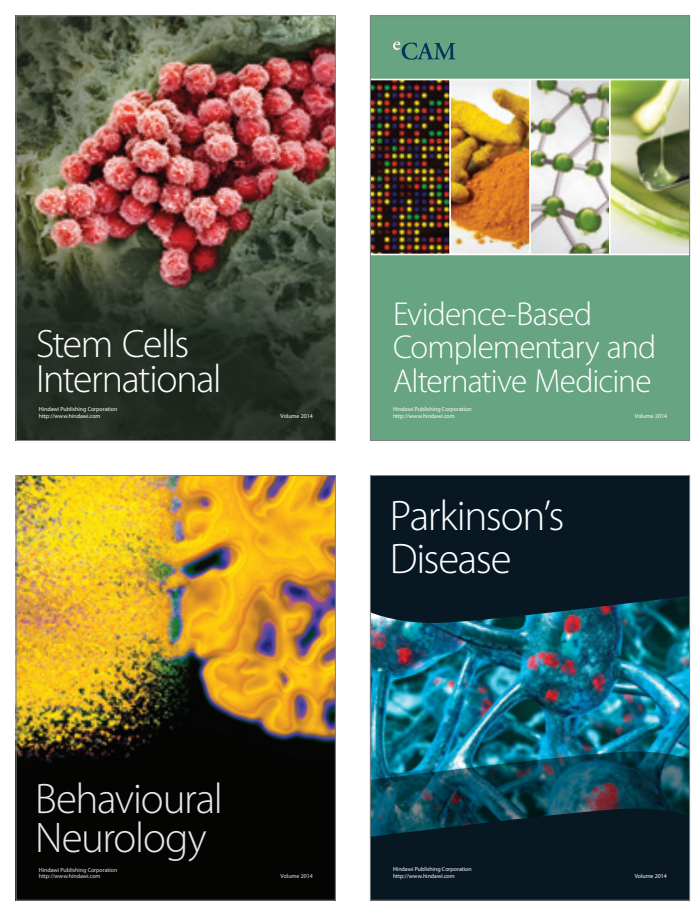

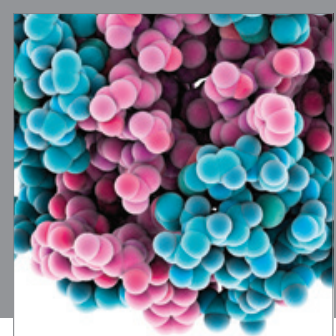

Journal of
Diabetes Research

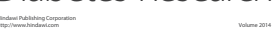

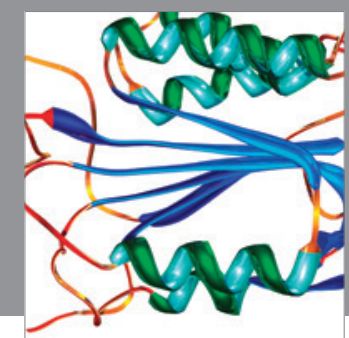

Disease Markers
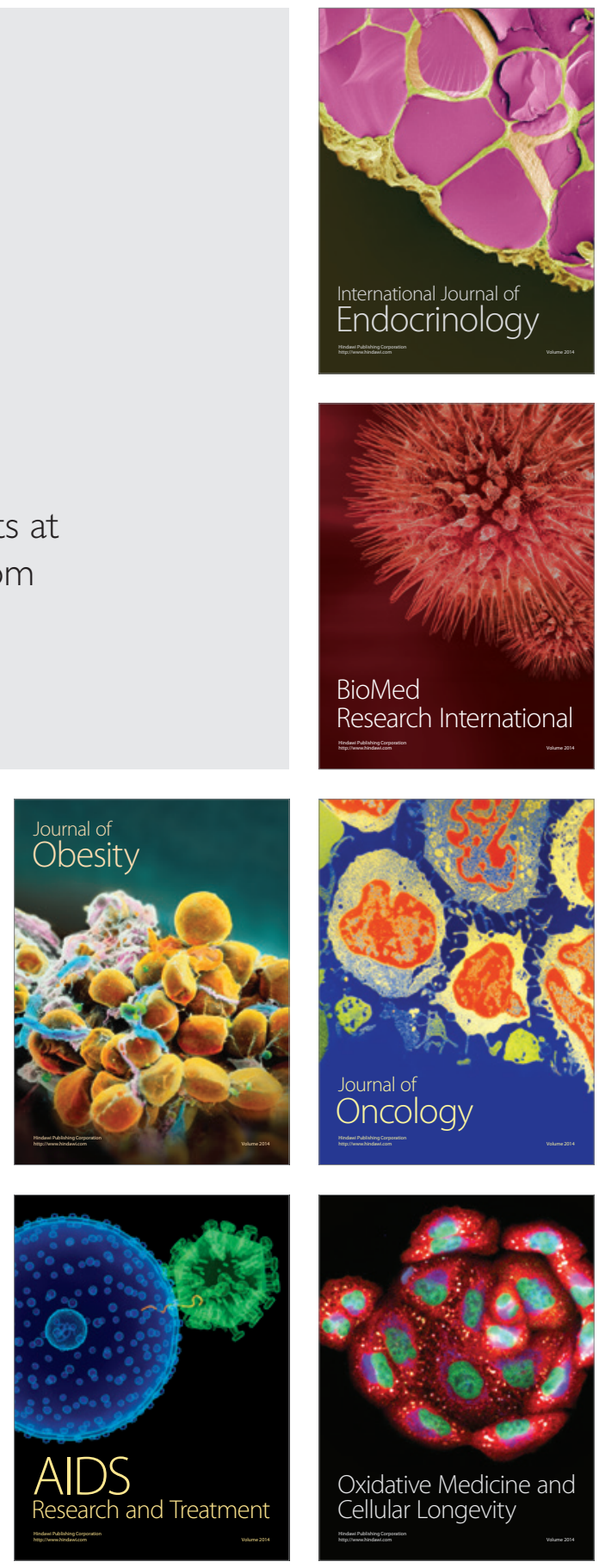